\title{
A Design of LPDA Antenna with Patch Dipoles for Airbone Communication
}

\author{
Ying Liu ${ }^{1, a}$, Yuwen Wang ${ }^{2, b}$, Yongwei $\mathrm{Li}^{2, \mathrm{c}}$ and Ting Yin ${ }^{2, \mathrm{~d}}$ \\ School of Aeronautics and Astronautics, University of Electronic Science and Technology of China, \\ Chengdu611731, China
}

\begin{abstract}
This article designs a Log-periodic Dipole Antenna(LPDA) for airborne communica-tion. This antenna replaces traditional dipoles with patch dipoles, the feeder adopt gradual changing structure, its working frequency is from $200 \mathrm{MHz}$ to $450 \mathrm{MHz}$.Use HFSS which is the special software for high-frequency electromagnetic to calculate, optimize and simulate. The simulation rsults are in good agreement with the test results. The designed antenna improves its flexibility and reduces profile, it provide a good method for the design of the airborne antenna.
\end{abstract}

\section{Introduction}

LPDA(Log Periodic Antenna) is proposed in 1957, it is a kind of directional antenna[1-6],LPDA is a $\mathrm{FI}$ (frequency independent)antenna, FI is that the antenna is transformed by a proportional factor $\tau$, the performance of antenna operating requency is equal to antenna operating frequency.LPDA has wider bandwith, which can be designed as 10:1(the ratio of the highest frequency to the lowest frequency),obviously,LPDA belongs to UWB(Ultra Wide Band)antenna[7].The profile of traditional LPDA[8] is relatively high,and its flexibility is not very ideal,but we can replace traditional dipoles with patch dipoles to research airborne antenna.Patch-dipoles LPDA is better to keep correspondence with the wing of aircraft,what's more, its electrical properties is less affected by the deformation of the wing.so we design a patch-dipoles LPDA applied to airborne antenna.

\section{The Design of Patch-dipoles LPDA}

A patched-dipoles LPDA operating at $200 \mathrm{MHz}-450 \mathrm{MHz}$ was designed for a gain of $10 \mathrm{~dB}$.the structure of LPDA[9] is affected by the combination of the scaling factor Tau $(\tau)$ and spacing factor $\operatorname{Sigma}(\sigma)[10]$, which can be calculated from carrel curves[11], $\tau$ corresponding to the best design is $0.95, \sigma$ is $0.17 . \alpha$ is 8.411 degree, $\mathrm{N}$ (the numbers of dipoles) is 17.From the operating principle of LPDA, we can know that for obtainning higher gain,we must add the number of dipoles,so we add four dipoles, the number of dipoles of LPDA is 21 .

The input impedence of LPDA is about $80 \mathrm{ohm}$, it is easy to match with coaxial cable which characteristic impedance is $75 \mathrm{ohm}$. In the field of military radio communication, the characteristic impedance of the mutual of each communication module is $50 \mathrm{ohm}$,obviously,LPDAwhich input impedance is $80 \mathrm{ohm}$ connecting with coaxial cable which characteristic impedance is $50 \mathrm{ohm}$ will

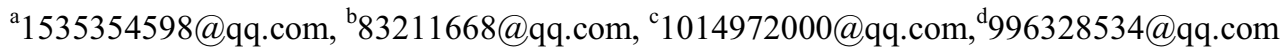


cause impedance adaptation and cause VSWR[12] too large,so we need to connect an impedance matching device between the feeder terminal of LPDA and coaxial cable, however, the antenna selected operate at $200 \mathrm{MHz}$ to $450 \mathrm{MHz}$, its belong to UWB antenna, it is difficult to design impedance matching device of UWB antenna system, besides, The introduction of he impedance matching device will not only cause the complex structure of the antenna,increase the size of antenna,but aslo bring a certain insertion loss, which affect the efficiency.for solving the difficult problem, the feeder of LPDA antenna we select adopt gradual changing structure,the width of LPDA in high frequency is 2 millimeter,the width of LPDA in low frequency is 20 millimeter,in this way,the input impedance of patch-dipoles LPDA is very close to $50 \mathrm{ohm}$, it match with coaxial cable well.

\section{The Structure and Results of LPDA In HFSS}

The structure of simulation of LPDA is showed in Fig.1.

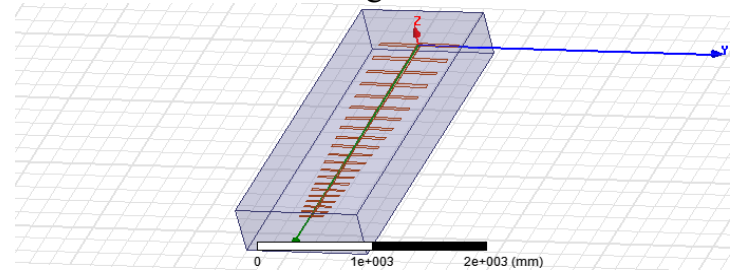

Figure 1. The structure of LPDA

By optimize, we can get the radiation patterns of LPDA antenna which dipoles are 21. The E-plane and H-plane radiation patterns of LPDA operating at $200 \mathrm{MHz}$ are showed in Fig.2 and Fig.3. The Eplane and H-plane radiation patterns of LPDA operating at $250 \mathrm{MHz}$ are showed in Fig.4 and Fig.5. The E-plane and H-plane radiation patterns of LPDA operating at $300 \mathrm{MHz}$ are showed in Fig. 6 and Fig.7. The E-plane and H-plane radiation patterns of LPDA operating at 350MHz are showed in Fig.8 and Fig.9. The E-plane and H-plane radiation patterns of LPDA operating at $400 \mathrm{MHz}$ are showed in Fig.10 and Fig.11. The E-plane and H-plane radiation patterns of LPDA operating at $450 \mathrm{MHz}$ are showed in Fig.12 and Fig.13.From the results of simulation,we can see that the biggest gain at $200 \mathrm{MHz}$ is $11.34 \mathrm{~dB}$, the biggest gain at $450 \mathrm{MHz}$ is $10.8 \mathrm{~dB}$, in the whole frequency band,LPDA can aslo get high gains.

\begin{tabular}{|c|c|c|c|}
\hline Name & Phi & Ang & Mag \\
\hline $\mathrm{m} 1$ & 0.0000 & 0.0000 & 11.3381 \\
\hline
\end{tabular}
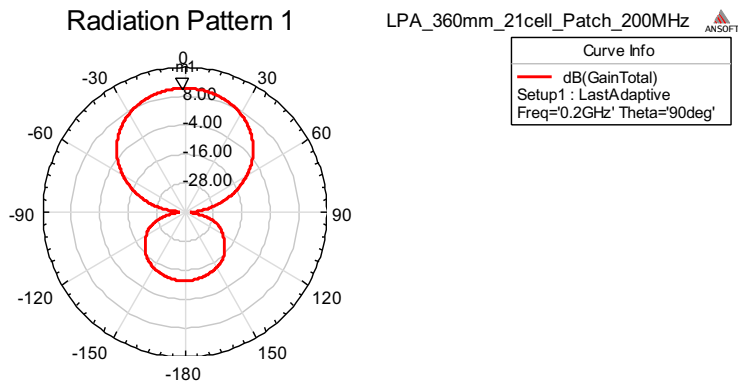

Figure 2. E-plane radiation patterns of LPDA ( $200 \mathrm{MHz})$ 


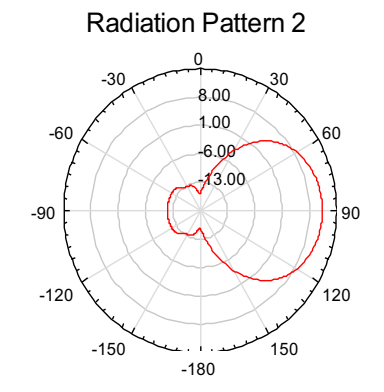

LPA_360mm_21cell_Patch_200MHz ANSAF

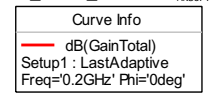

Figure 3. H-plane radiation patterns of LPDA( $200 \mathrm{MHz})$

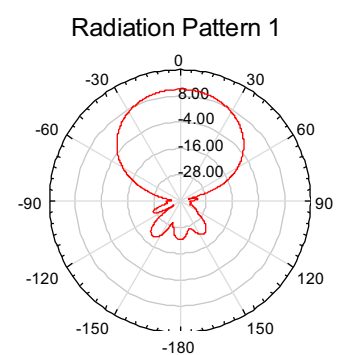

Figure 4. E-plane radiation patterns of LPDA ( $250 \mathrm{MHz})$

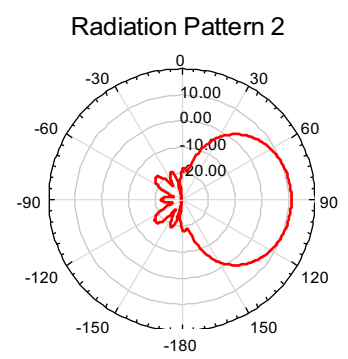

Figure 5. H-plane radiation patterns of LPDA $(250 \mathrm{MHz})$

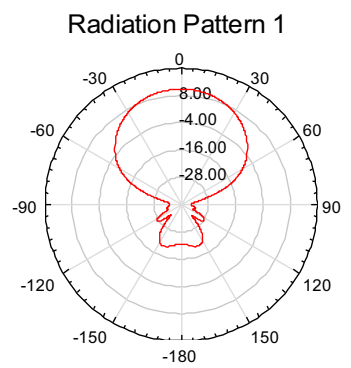

LPA_360mm_21cell_Patch_300MHz ANSAOF

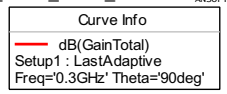

Figure 6. E-plane radiation patterns of LPDA $(300 \mathrm{MHz})$

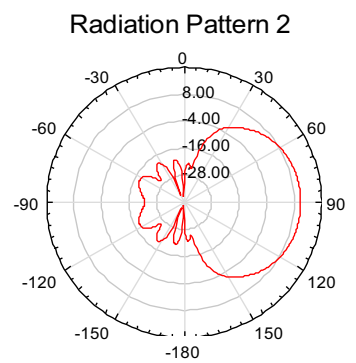

LPA_360mm_21cell_Patch_300MHz ANsAo

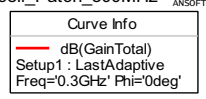

Figure 7. H-plane radiation patterns of LPDA( $300 \mathrm{MHz})$ 


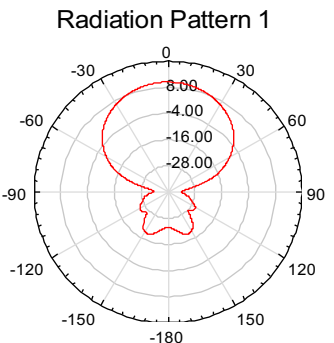

LPA 360mm 21cell Patch $350 \mathrm{MHz}$

Figure 8. E-plane radiation patterns of LPDA $(350 \mathrm{MHz})$
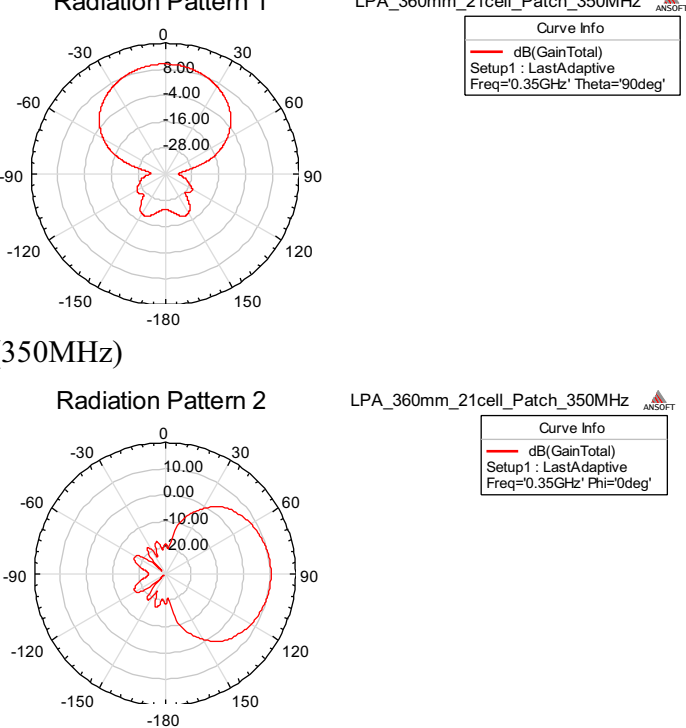

LPA 360mm 21cell_Patch $350 \mathrm{MHz}$

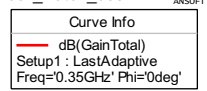

Figure 9. H-plane radiation patterns of LPDA $(350 \mathrm{MHz})$
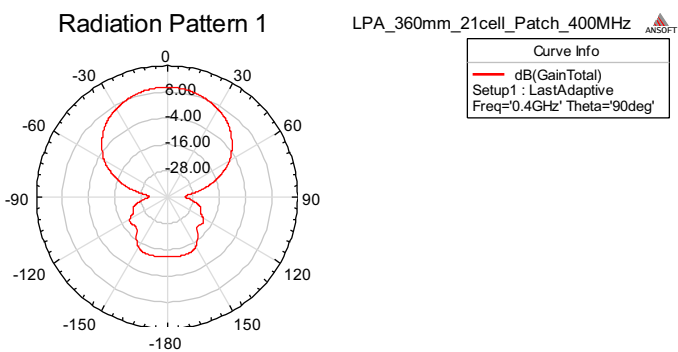

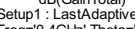
$\begin{array}{ll} & \\ & \end{array}$

Figure 10. E-plane radiation patterns of LPDA( $400 \mathrm{MHz})$

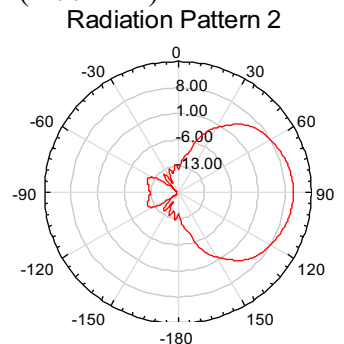

LPA_360mm_21cell_Patch_400MHz AN50

\begin{tabular}{|c|}
\hline \multicolumn{1}{c|}{ Curve Info } \\
dB(GainTotal) \\
\hline Setup1: LastAdaptive \\
Freq $=0$ ' $0.4 G$ '
\end{tabular}
Setup 1: LastAdaptive
Freq='0.4GH२' Phi='0deg

Figure 11. H-plane radiation patterns of LPDA $(400 \mathrm{MHz})$

\begin{tabular}{|c|c|c|c|}
\hline Name & Phi & Ang & Mag \\
\hline $\mathrm{m} 1$ & 0.2000 & 3.0000 & 10.8259 \\
\hline
\end{tabular}

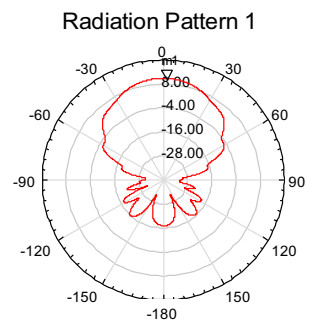

LPA_360mm_21cell_Patch_450MHz Ansof

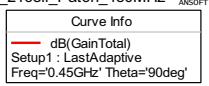

Figure 12. E-plane radiation patterns of LPDA $(450 \mathrm{MHz})$ 

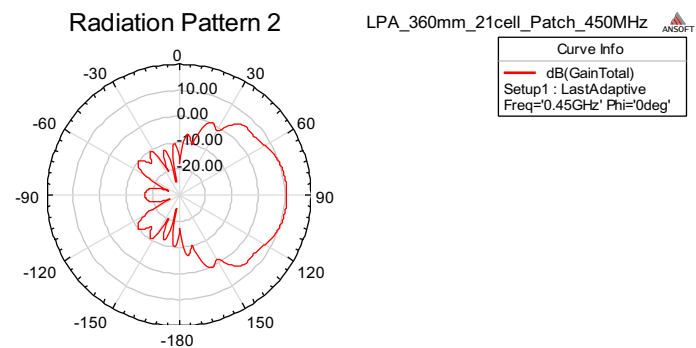

Figure 13. H-plane radiation patterns of LPDA( $450 \mathrm{MHz})$

\section{The Physical Design of LPDA}

Since the size of LPDA operating at $200 \mathrm{MHz}$ to $450 \mathrm{MHz}$ is too large, it is not easy to test its electrical properties,for solving this problem,we adopt scaling shrunk simulation measuring method,scaling factor is 4 , the size of antenna for the test is $1 / 4$ of the original LPDA antenna,in this way,we can solve many problems caused by the too large size of antenna when we measure antenna.when the scaling factor is 4 , the operating frequency of LPDA will be changed into $800 \mathrm{MHz}$ to $1800 \mathrm{MHz}$. The physical LPDA is showed in Fig.14.In the laboratory of College of Aeronautics and Astronautics of UESTC, we use Vector Network Analyzer (Type:N5242A) designed by Alilent to measure the scaling LPDA.The reflective characters of LPDA measured is showed in Fig.15. The measurment of antenna is showned inFig.16.From Fig.15,we can see that within the frequency band of $600 \mathrm{MHz}$ to $2000 \mathrm{MHz}$, the reflective characters of antenna(scaling factor is 4) is below -10dB,this shows that the antenna(actual size) we designed operating frequency at $150 \mathrm{MHz}$ to $500 \mathrm{MHz}$, its reflective characters is below $-10 \mathrm{~dB}$ too. The actual bandwidth of LPDA we designed $(150 \mathrm{MHz}-500 \mathrm{MHz})$ is better than the original bandwidth $(200 \mathrm{MHz}-450 \mathrm{MHz})$.

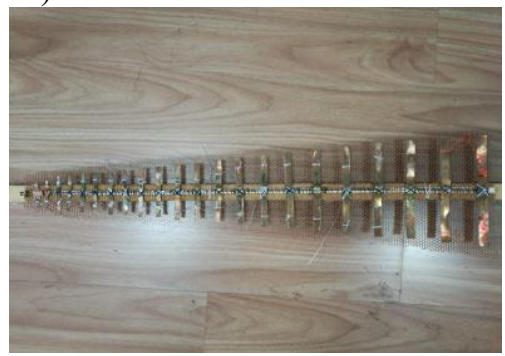

Figure 14. The physical LPDA

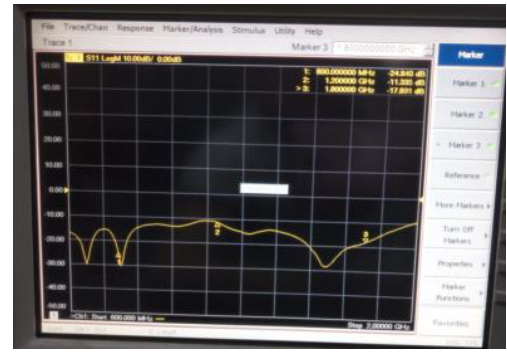

Figure 15. The reflective characters of LPDA 
Figure 16. The measurment of antenna

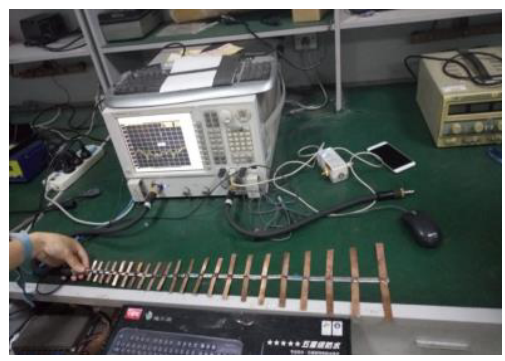

\section{Summary}

The patch-dipoles LPDA we designed operating at $200 \mathrm{MHz}$ to $450 \mathrm{MHz}$ has wide bandwidth, high gain, low profile, good flexibility and light weight etc. It provides a reliable choice for airborne antenna, particularly in the antenna of solar powered warning drones in Near Space.

\section{References}

1. DuHamel R H, Ore F. Logarithmically Periodic Antenna Designs [C]. IRE International Coventional Record, 2001(6): 139-151.

2. Nguyen C. A Wide-band Microstrip-fed Log-periodic Antenna [C]. Antennas and Propagation Society International Symposium, 2006(1):33-36.

3. Mohammad M T. Wideband Planar Log -periodic Antenna [C]. Antenna technology: Small and Smart Antennas Metamaterials and Applications, 2007.IWAT '07. International Workshop on, 2007:331-334.

4. Orlob C,Dao Q H, Kornek D. Dual-polarized Log -periodic Antenna on a Conical MID Substrate[C]. Antennas and Propagation(EUCAP), Proceedings of the $5^{\text {th }}$ European Conference on, 2011:361-364.

5. D.E.Isbell. Log-periodic dipole arrays[J]. IRE Trans. Antennas Propag., 2011, AP-8: 260-267.

6. R.L.Carrel. The design of log-periodic antennas[J]. IRE Int.Conent.. Rec., 2010, 9(1): 61-75.

7. Praveen Nartam, Abhijeet Gulawani, and Subhajit Chatterjee, "Design of an Ultra wide Band antenna using defected ground structure,"ICCPEIC, pp. 75-79, 2016.

8. Zou Aihua and Xu Changwu, "A small dual stacked log periodic dipole array antenna(LPDA) with improved gain of low frequency for immunity,"APEMC,pp.208-211,2015.

9. Wang Zhefei and $\mathrm{Fu}$ Jiahui, "A design of miniaturization LPDA antenna with ultra sideband,"2015 IEEE 6th International Symposium on Microwave,Antenna,Propagation,and EMC Technologies,pp819-821,2015.

10. Jerin Jose,Tessa Mathew,Annu Thomas,Haripriya N,Mahima Cherian, and D.D.Krishna, “A Cost Effective Hybrid-Log Periodic Dipole Antenna,"2015 Fifth International Conference on Advances in Computing and Communications,pp263-265,2015.

11. Carrel,R.1, "Analysis and design of the log-periodic dipole antenna,"Technical Report,No.52,Antenna Laboratory,University of Illinois 1961.

12. T,Taniguchi and T.Kobayashi, "An omnidirectional and low-VSWR antenna for ultra-wideband wireless systems",Radio andWireless and Conference,pp.145-148,2002. 